\title{
From ab-initio calculations to multiscale design of Si/C core-shell particles for Li-ion anodes
}

Maria E. Stournara1*, Yue Qi2,3*, Vivek B. Shenoy1,4*

Corresponding author: maria_stournara@brown.edu

1 School of Engineering, Brown University, Providence, RI 02912

2Department of Chemical Engineering and Materials Science, Michigan State University, East Lansing, MI,4882

3General Motors Global Research \& Development Center, 30500 Mound Road, Warren, MI 48090

4Departments of Materials Science and Engineering \& Mechanical Engineering and Applied Mechanics, University of Pennsylvania, Philadelphia, PA 19104 


\section{Supporting Information}

\section{Evaluating Surface Stability of $\mathrm{C}$ at the presence of $\mathrm{Li}$ and $\mathrm{Si}$}

\section{Diamond (111) Surface Reconstruction}

We combined results from $a b$-inito with concepts from thermodynamics to predict the structural evolution of the a-LiSi/a- $\mathrm{Li}_{0.375} \mathrm{C}_{6}$ interface upon lithiation. We evaluated the stability of the a-C free surface at the presence of $\mathrm{Li}$ and $\mathrm{Si}$ by designing clean (111) diamond surfaces and passivating them with mixed $\mathrm{Li}$ and $\mathrm{Si}$ to generate nonstoichiometric $\mathrm{Li}$ - and $\mathrm{Si}$-terminated surfaces. Using the calculated surface energies, we constructed a surface phase diagram that allowed us to gauge thermodynamic stability. The slab models were designed and the calculations were performed as described in Sec. II and surface energies of diamond (111) surfaces for 5-8 layers of slabs were calculated to determine the necessary slab thickness according to the bulk structure. The surface energies for the clean diamond surface were calculated using Boettger's method [1]

$$
E_{s}(n)=\frac{1}{2 A}\{E(n)-n[E(n)-E(n-1)]\}
$$

where $E(n)$ is the energy of the $n$ layer slab and $A$ is the area of the surface. The results are reported in Table 1. Because there are four atomic positions at the (111) surface, there are also four $\mathrm{Si}$ atoms at the fully Si-terminated surface and four $\mathrm{Li}$ at the fully $\mathrm{Li}$-terminated surface. Replacing the Li atoms, one by one, with $\mathrm{Si}$ atoms resulted in various surface concentrations, such as 0.25, 0.5, 0.75 and full Si-terminated surfaces (Fig. 1). As the surfaces contained chemical species that differed from the bulk elements of the structure, the surface energies were defined according to the chemical potentials of existing species. Therefore, the surface energy, $\mathrm{E}_{\mathrm{s}}$, was defined as a function of chemical potentials of $\mathrm{Li}$ and $\mathrm{Si}$ and the corresponding equation is given by

$$
E_{s}=\frac{1}{2 A}\left[E^{s l a b}-\left(n_{c} \mu_{c}+n_{L i} \mu_{L i}+n_{S i} \mu_{S i}\right)\right]
$$

where $n_{C}, n_{L i}$, and $n_{S i}$ are the number of C, Li, and Si atoms in the slab, $\mu_{C}, \mu_{L i}$, and $\mu_{S i}$ are the chemical potentials of $\mathrm{C}, \mathrm{Li}$, and $\mathrm{Si}$, respectively, and $\mathrm{E}^{\text {slab }}$ is the energy of the slab. Note that the chemical potentials of $\mathrm{Li}$ and $\mathrm{Si}$ depend on the $\mathrm{Li}_{\mathrm{x}} \mathrm{Si}$ that the surface is in equilibrium with. 
To determine which phase had the lower surface energy, and hence, was more stable for any given chemical potential of hydrogen and fluorine, a surface phase diagram was constructed that shows the predominant areas of the stable structures. The surface termination phase diagram constructed is shown in Fig., which is obtained by projecting the lowest surface free energies on the $\mu_{L i}-\mu_{L i}^{o}$ and $\mu_{S i}-\mu_{S i}^{o}$ planes. According to Fig. 2, the Si-terminated surface is more stable in a much larger phase space than the Li-terminated one. At zero chemical potential of $\mathrm{Li}$ and $\mathrm{Si}$, which correspond to $\mathrm{Li}$ and $\mathrm{Si}$ bulk phases, the stable surface is $0.75 \mathrm{Si}$-terminated. Increasing the Si concentration makes a fully Si-terminated surface formation more favorable. Similarly, a Li-terminated surface can be achieved by increasing the chemical potential of Li. The desired surface composition can be adjusted by varying the composition of $\mathrm{Li}, \mathrm{Si}, \mathrm{LiSi}$ and $\mathrm{Li}_{1.71} \mathrm{Si}$. However if the diamond (111) surface is exposed to $\mathrm{Si}, \mathrm{LiSi}$ and $\mathrm{Li}_{1.71} \mathrm{Si}$, it will always be fully Si terminated, and a fully Li-terminated surface that is exposed to a Si-rich environment, will produce a $0.75 \mathrm{Si}$-terminated surface, indicating a change during surface modification. This

finding provides an essential understanding of the surface chemistry of carbon and is of great significance for the study of the a-LiSi/a- $\mathrm{Li}_{0.375} \mathrm{C}_{6}$ interface. As $\mathrm{C}$ bonds preferentially with $\mathrm{Si}$ rather than $\mathrm{Li}$, $\mathrm{Li}$ will not segregate at the a-LiSi/a- $\mathrm{Li}_{0.375} \mathrm{C}_{6}$ interface. This statement is further discussed in terms of the electronic structure of the nonstoichiometrically terminated C-surfaces in the following section.

\section{The Bonding Nature of the Diamond Surface at Various Terminations}

The reason for the increased presence of Si atoms on the diamond (111) surface lies in the bonding nature of the nonstoichiometrically terminated surfaces. Electron density distributions of the surfaces were mapped to determine the surface area coverage of Si termination, as shown in Fig. 3. The electron density coverage increases with an increase in the $\mathrm{Si}$ content at the surface. The fully Li-terminated surface has almost covered the entire diamond surface compared with the fully Si-terminated surface, whereas there was not a significant charge accumulation on the fully Si-terminated surface. Bader charge analysis was used to partition electrons and atoms, and the net charges on the Li- and Si-terminated diamond (111) surfaces with reference to $\mathrm{Li}$ and $\mathrm{Si}$ bulk structures and perfect diamond appear in Table 2. The $\mathrm{C}$ atoms at the center of the slab were almost neutral $\left(\mathrm{e}^{-}<0.007\right)$, which is comparable to the zero charge determined for perfect diamond. This observation verifies that the slab model is sufficiently thick 
to maintain the bulk properties of diamond at the slab center. The "diffuse" signature of the pure $\mathrm{Li}$ termination was attributed to the metallic nature of $\mathrm{Li}$ : Though every $\mathrm{Li}$ atom donated approximately $0.7-0.8$ electrons to the each $\mathrm{C}$ surface atom, only 0.2 of this charge contributed in the formation of the Li-C bond, leaving the remaining 0.5-0.6 cover the diamond surface in the form of an electron cloud (light blue color in the ELF plot). On the contrary, in all Si-containing surfaces, the Si atom took charges from the $\mathrm{C}$ atom underneath, resulting in negatively $0.44-0.48$ charged $\mathrm{Si}$ and positively $0.46-0.5$ charged $\mathrm{C}$ atoms. This ionic character of the $\mathrm{C}-\mathrm{Si}$ bond contributed to an increase in the stability of the Si-terminated surface. This finding is in excellent agreement with the large electronegativity difference, 1.57 , between $\mathrm{Si}$, with an electronegativity of 1.9 , and $\mathrm{C}$, with an electronegativity of 2.55 . The difference in electronegativity between $\mathrm{C}$ and $\mathrm{Li}$ is as small as 0.65 , suggesting the $\mathrm{C}$ - $\mathrm{Li}$ bond has a more diffuse character, with the electrons involved being delocalized along the surface, resulting in a weaker bond.

Table 1: Surface energy convergence of clean diamond (111) surfaces according to number of layers (n) in the surface structure.

\begin{tabular}{cccc}
\hline \hline $\mathbf{n}$ & No. of atoms & $\mathbf{E}_{\mathbf{s}}(\mathbf{n})(\mathbf{e V} / \mathbf{a t o m})$ & $\mathbf{E}_{\mathbf{s}}(\mathbf{n})\left(\mathbf{J} / \mathbf{m}^{\mathbf{2}}\right)$ \\
\hline 6 & 48 & 0.6647 & 10.6352 \\
7 & 56 & 0.6643 & 10.6293 \\
8 & 64 & 0.6642 & 10.6287 \\
9 & 72 & 0.6658 & 10.6536 \\
\hline \hline
\end{tabular}

Table 2: Bader charge analysis of Li- and Si-terminated diamond (111) surfaces. Values show the amount of charge transferred, with a positive value representing charge accumulation and a negative value representing charge depletion on the corresponding atom.

\begin{tabular}{cccccc}
\hline Surface Term. & $\mathbf{L i}$ & $\mathbf{C}$ below Li & Si & C below Si & Bulk C \\
\hline $\mathrm{Li}_{4}$ & 0.737 & -0.294 & -- & --- & -0.006 \\
$\mathrm{Li}_{3} \mathrm{Si}_{1}$ & 0.781 & -0.248 & 0.480 & -0.468 & 0.002 \\
$\mathrm{Li}_{2} \mathrm{Si}_{2}$ & 0.794 & -0.221 & 0.457 & -0.474 & -0.001 \\
$\mathrm{Li}_{1} \mathrm{Si}_{3}$ & 0.819 & -0.210 & 0.447 & -0.472 & 0.002 \\
$\mathrm{Si}_{4}$ & --- & --- & 0.453 & -0.506 & 0.007 \\
\hline \hline
\end{tabular}



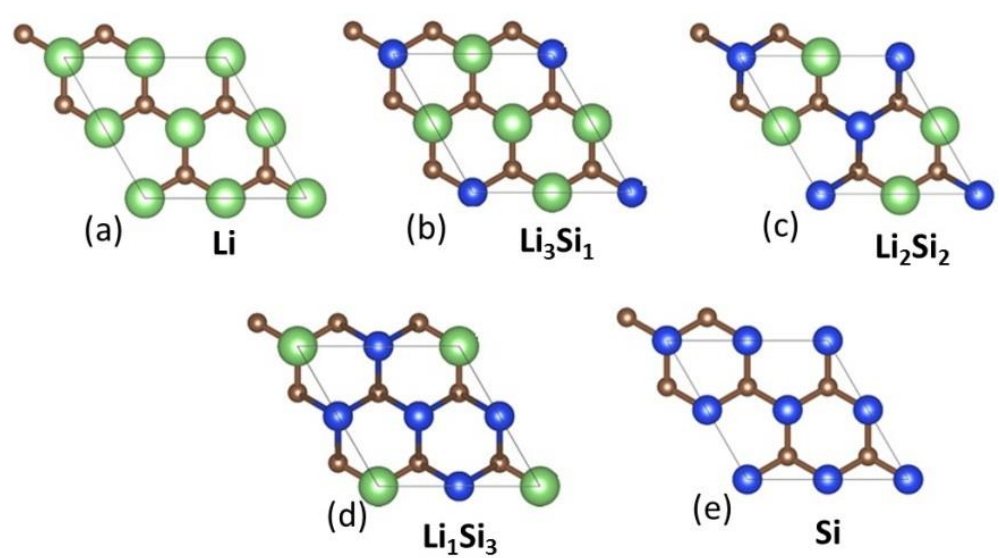

Fig. 1: Surface termination of diamond (111) surfaces: (a) Li-terminated, (b-d) mixed terminated surfaces, (e) Si-terminated

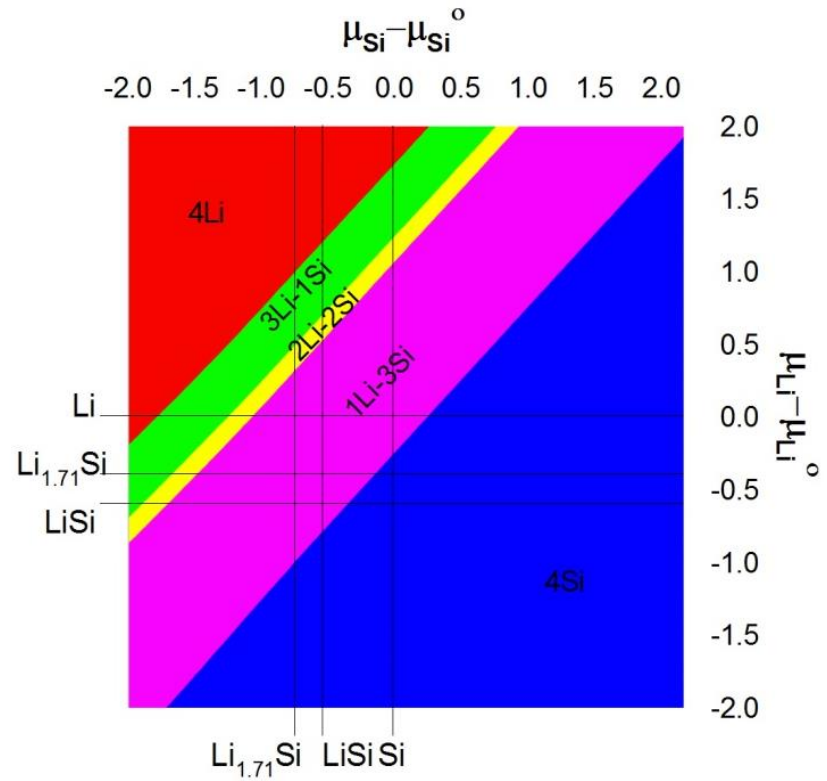

Fig. 2: Surface phase diagram od stable Li- and Si- terminated diamond (111) structures. The graphs is the projection of the prevalent surface energies on the $\Delta \mu_{\mathrm{Li}}$ and $\Delta \mu_{\mathrm{Si}}$ plane.

Terminations for a particular $\mathrm{Li}, \mathrm{Si}$ and $\mathrm{LiSi}_{2}$ composition are shown as a function of the bulk $\mathrm{Li}$ $\left(\mu_{\mathrm{Li}}-\mu_{\mathrm{Li}}{ }^{\mathrm{o}}=1.977 \mathrm{eV} /\right.$ atom $)$ and $\mathrm{Si}\left(\mu_{\mathrm{Si}}-\mu_{\mathrm{Si}}{ }^{\mathrm{o}}=1.023 \mathrm{eV} /\right.$ atom $)$. 

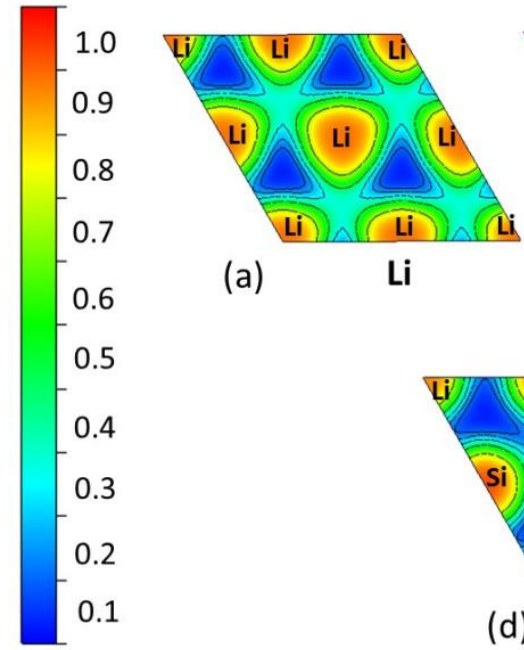

(a)

Li

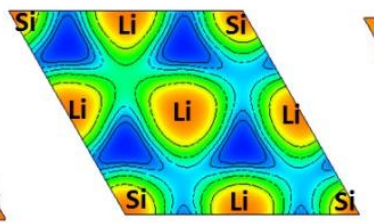

(b) $\quad \mathrm{Li}_{3} \mathrm{Si}_{1}$

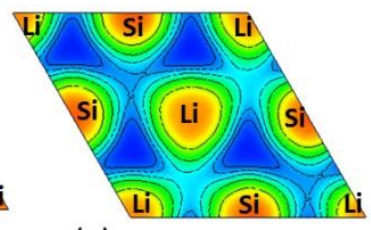

(c) $\quad \mathrm{Li}_{2} \mathrm{Si}_{2}$

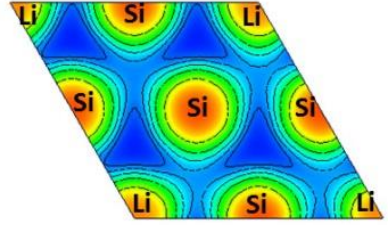

(d)

$\mathrm{Li}_{1} \mathrm{Si}_{3}$

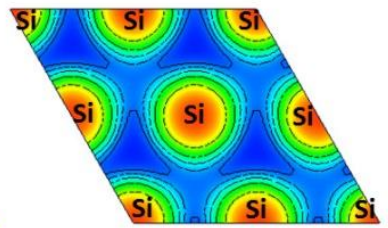

(e) $\quad \mathrm{Si}$

Fig. 3: ELF plots of Li- and Si-terminated diamond (111) surfaces showing the surface coverage of different terminations: (a) Li-terminated, (b-d) mixed terminated surfaces, (e) Si-terminated.

1. Boettger, J. C., Nonconvergence of surface energies obtained from thin-film calculations. Physical Review B, 1994. 49(23): p. 16798-16800. 